\title{
Latest developments in the field of stem cell research and regenerative medicine compiled from publicly available information and press releases from nonacademic institutions in November 2018
}

\author{
Dusko llic*,1 \& Shi Yin Ang ${ }^{2}$ \\ ${ }^{1}$ Stem Cell Laboratories, Guy's Assisted Conception Unit, Department of Women \& Children's Health, Faculty of Life Sciences \& \\ Medicine, King's College London, London, UK \\ ${ }^{2} \mathrm{MSc}$ Stem Cell \& Regenerative Therapies: from Bench to Market, Faculty of Life Sciences \& Medicine, King's College London, \\ London, UK \\ *Author for correspondence: dusko.ilic@kcl.ac.uk
}

First draft submitted: 10 January 2019; Accepted for publication: 12 February 2019; Published online:

26 March 2019

\section{Business development}

Collaborations, partnerships \& alliances

License agreement: Cabaletta \& UPenn

Cabaletta Bio (PA, USA; www.cabalettabio.com) has signed an exclusive license agreement and executed two multiyear sponsored research agreements with the University of Pennsylvania (PA, USA; www.upenn.edu) for the discovery and development of engineered T-cell therapy products for B-cell-mediated autoimmune disease. The engineered T-cell technology utilizes chimeric autoantibody receptors (CAARs) to bind and destroy only diseasecausing B cells, while sparing the normal B cells which are essential for human health. In addition, the Company has entered into a master services agreement with Penn that will allow it to enter into agreements for scientific, clinical and manufacturing expertise to develop a first CAAR product for mucosal pemphigus vulgaris [1]. The Company's lead product is designed to selectively eradicate B cells that produce autoantibodies to desmoglein 3 (DSG3), which are necessary to cause mucosal pemphigus vulgaris. The Company recently completed a US $\$ 38$ million Series A financing to advance this lead asset, DSG3-CAART, into clinical development.

\section{License agreement: ERS Genomics \& DefiniGEN}

ERS Genomics (Ireland; www.ersgenomics.com) and DefiniGEN (UK; www.definigen.com) have announced a license agreement to provide DefiniGEN with access to ERS Genomic's CRISPR/Cas9 genome editing technology patents. DefiniGEN will combine their best-in-class induced pluripotent stem cell (iPSC) differentiation platform with CRISPR/Cas9 technology to generate a range of innovative preclinical human models.

\section{Partnership agreement Vineti \& Tessa}

Vineti (CA, USA; www.vineti.com) has announced a partnership with Tessa Therapeutics (Singapore, www.tess atherapeutics.com) to advance and scale personalized cell therapies for solid tumors in Asia and worldwide. Tessa Therapeutics (Tessa) is a developer of personalized T-cell therapies for solid tumors, including head and neck cancers and cervical cancer. Vineti provides the digital software platform to align and manage the complex personalized cell therapy process, safely and efficiently. The goal of the partnership is to scale Tessa's therapies worldwide in a safe and efficient manner, supporting both late clinical stage therapies approaching commercialization and early-phase therapies progressing through various clinical stages. Tessa brings international expertise through both its Asia operations and its global treatment network. 


\section{Achievements}

HemaCare

HemaCare (CA, USA; www.hemacare.com) has announced their involvement as a provider of leukapheresis process development material for $100 \%$ of the current US FDA approved immunocellular therapies, Kymriah ${ }^{\circledR}$ (Novartis; Switzerland; www.novartis.com), Yescarta ${ }^{\circledR}$ (Kite, a Gilead Company; CA, USA; www.kitepharma .com) and PROVENGE ${ }^{\circledR}$ (sipuleucel-T; Dendreon; CA, USA; www.dendreon.com). During the development process, HemaCare worked collaboratively with each company to source healthy donors per project requirements. All leukapheresis material was collected and shipped following stringent standards as developed by the AABB and accepted by the FDA. In some cases, HemaCare performed further processing by splitting collections for comparability runs, freezing material or adding isolated cells to mimic patient collection material.

\section{Clinical trials \\ Asterias}

Asterias Biotherapeutics (CA, USA; https://asteriasbiotherapeutics.com) has enrolled and dosed the fourth subject in the first-in-human Phase I clinical trial of VAC2 in the UK. This is the first patient enrolled and dosed at Queen Elizabeth Hospital, Birmingham, UK. This initial clinical trial, which is being sponsored, managed and funded by Cancer Research UK, will examine the safety and tolerability of VAC2 in non-small-cell lung cancer (NSCLC) as the study's primary end points. Secondary and tertiary end points of the study include evaluations of the immunogenicity of VAC2 in NSCLC. VAC2 is an innovative immunotherapy product that contains mature dendritic cells derived from iPSCs. These allogeneic VAC2 cells are engineered to express a modified form of telomerase, a protein widely expressed in tumor cells, but rarely found in normal cells. The modified form of telomerase invokes enhanced stimulation of immune responses to the protein. Similar to an earlier, Asteriassponsored, hematological cancer program using an autologous approach, the VAC2 dendritic cells instruct the immune system to generate responses against telomerase and, through this mechanism, target tumor cells. VAC2's mode of action is complementary to and potentially synergistic with other immune therapies such as checkpoint inhibitors or other immune pathway inhibitors.

\section{BioCardia}

BioCardia (CA, USA; www.biocardia.com) has announced 12-month data for the CardiAMPTM Heart Failure Trial (https://clinicaltrials.gov; ID: NCT02438306) studying the investigational CardiAMP Cell Therapy System in adult patients experiencing heart failure following a heart attack.

CardiAMP cell therapy uses autologous bone marrow cells delivered to the heart in a minimally invasive, catheterbased procedure to potentially stimulate the body's natural healing response. The CardiAMP Heart Failure Trial is the first multicenter clinical trial of a stem cell therapy to prospectively screen for stem cell therapeutic potency in order to improve patient outcomes.

At the primary end point of exercise capacity at 12 months, the 10-patient roll-in cohort of the trial showed clinically meaningful improvement, walking an average of 46.4 meters more than baseline $(\mathrm{N}=10)$, although the improvement was not considered statistically significant $(\mathrm{p}=0.063)$. Eight of the 10 patients experienced improvement in their exercise capacity. This improvement is more than triple the average improvement over baseline reported in the CardiAMP-treated arm of the Phase II TAC-HFT-MNC trial, and greater than the average improvement seen in a number of pivotal trials for implantable cardiac resynchronization therapy to treat heart failure.

In the secondary efficacy end point of quality of life, patients showed a clinically meaningful improvement of 9.8 points relative to baseline, which was not statistically significant $(p=0.331)$ in the small cohort. Seven of the 10 patients reported better quality of life after CardiAMP treatment. This was a greater improvement over baseline than was seen in the Phase II TAC-HFT-MNC trial of CardiAMP therapy. The secondary efficacy end points of superiority relative to major adverse cardiac events (MACE) and survival were not possible to assess in this roll-in cohort as there is no control arm specific to this cohort. However, there were no treatment emergent major adverse cardiac events in this group at 30 days, while there was one MACE event due to a hospitalization at 9 months. All MACE has not been adjudicated at this time. All patients from this cohort were alive and out of hospital at 12 months. 


\begin{abstract}
Japan
Japanese neurosurgeons have implanted 'reprogrammed' stem cells into the brain of a patient with Parkinson's disease for the first time. The condition is only the second for which a therapy has been trialed using induced iPSCs. Scientists at Kyoto University use the technique to transform iPSCs into precursors to the neurons that produce the neurotransmitter dopamine. The team will observe him for 6 months and, if no complications arise, will implant another 2.4 million dopamine precursor cells into his brain. The team plans to treat six more patients with Parkinson's disease to test the technique's safety and efficacy by the end of 2020.

In unrelated news, Keio University gave a tentative endorsement for the plan to conduct the world's first treatment of patients who have sustained spinal cord injuries using iPSCs.
\end{abstract}

\title{
Kolon TissueGene
}

Kolon TissueGene (Korea; www.tissuegene.com/en_US) has dosed its first patient in its pivotal US Phase III clinical trials for Invossa, a cell and gene therapy in development for knee osteoarthritis. Kolon TissueGene is conducting pivotal Phase III trials for US approval of Invossa for knee osteoarthritis. The pivotal Phase III trials for US approval of Invossa will enroll close to 1020 patients in approximately 60 clinical sites across USA. The trial investigators include orthopedic surgeons, rheumatologists and pain specialists. During the trial, the company will assess pain and function end points as well as MRI, x-ray and liquid biomarkers. In addition to demonstrating significant improvements in pain relief and function, the trials are designed to show structural benefits, including a delay in disease progression, and if successful could achieve a Disease Modifying Osteoarthritis Drug or 'DMOAD' label claim. Such an indication by the FDA would be a first for any osteoarthritis drug approved in USA. Details on the study can be found at: www.clinicaltrials.gov (ID: NCT03203330).

\section{Mesoblast}

Mesoblast (Australia; www.mesoblast.com) has announced that results from a 159-patient randomized, shamcontrolled Phase II trial in end-stage heart failure patients implanted with a left ventricular assist device showed that Mesoblast's allogeneic cell therapy candidate MPC-150-IM achieved significant reduction in major gastrointestinal (GI) bleeding episodes and related hospitalizations, a complication affecting up to $40 \%$ of left ventricular assist device recipients. Over the 6-month observation period, treatment with MPC-150-IM was associated with the following results:

- Significant reduction in cumulative incidence of major GI bleeding events by $48 \%$, from $33 \%$ in controls to $17 \%(p=0.02)$,

- Significant reduction in rate of major GI bleeding events by $76 \%$, from $15.9 / 100$ patient months to 3.8/100 patient months $(\mathrm{p}<0.001)$,

- Significant reduction in rate of hospitalization for GI bleeding, a major cause of hospital readmissions, by $65 \%$, from $0.21 / 100$ patient months to $0.07 / 100$ patient months $(\mathrm{p}=0.03)$,

- No patients experienced a safety-stopping event for the trial.

\section{SanBio}

The SanBio Group (Japan; www.sanbio.com) has announced that the Phase II STEMTRA trial using SB623 cells for the treatment of patients with traumatic brain injury, met its primary end point. This STEMTRA study (www.clinicaltrials.gov ID: NCT02416492) showed that the traumatic brain injury patients with chronic motor deficits treated with SB623 cells demonstrated a statistically significant improvement in their motor function compared with the control group, based on the Fugl-Meyer Motor Scale. The study met its primary end point, with SB623 patients achieving an average 8.7 point improvement from baseline in the Fugl-Meyer Motor Scale, versus 2.4 in the control group, at 24 weeks. SB623 cells are expanded from marrow stromal cells transfected with a Notch intracellular domain expressing plasmid. SB623 cells resemble the parental marrow stromal cells with respect to morphology and cell surface markers, despite their expansion in culture. The safety data showed that SB623 was well tolerated and no new safety signals were identified.

\section{Sorrento}

Sorrento Therapeutics (CA, USA; www.sorrentotherapeutics.com) has announced that the first patients were dosed in a Phase I study to evaluate the safety and efficacy of CD38 CAR-T therapy in relapsed or refractory multiple 
myeloma patients at two clinical sites - University of Pennsylvania (PA, USA; www.upenn.edu) and Roger Williams Medical Center (RI, USA; www.rwmc.org). The CD38 CAR-T cells manufactured at both Sorrento cGMP facilities in California and Rhode Island met all release specifications and were used in the study. This study is the first US-based clinical trial targeting CD38 using an autologous CAR-T-cell therapy. Details on the study can be found at: www.clinicaltrials.gov (ID: NCT03464916).

\section{SpinalCyte}

SpinalCyte (TX, USA; www.spinalcyte.com), a company focused on regrowth of the spinal disc nucleus using its universal donor product, CybroCell ${ }^{\mathrm{TM}}$, has announced that a single injection of modified human dermal fibroblasts, resulted in significant improvements of disc height and pain reduction 12 months after injection of the cell therapy for patients with degenerative disc disease. The trial assessed patients using the Oswestry disability index, visual analog scale and disc height via MRI scans. Using composite scoring, $54 \%$ of the treatment arm patients met all three end points as compared with only $17 \%$ with the placebo $(p=0.0003)$. Over $84 \%$ of patients in the treatment group of CybroCell ${ }^{T M}$ or CybroCell with platelet-rich plasma had an increase or no change in disc height compared with $25 \%$ for the placebo saline injection. On average, treatment group patients showed a $59 \%$ improvement in Oswestry Disability Index scores, compared with the $22 \%$ improvement in the placebo group.

\section{Regulations, approvals, acquisitions... Green light Fate Therapeutics}

Fate Therapeutics (CA, USA; www.fatetherapeutics.com) has announced that the US FDA has allowed its Investigational New Drug (IND) Application for FT500, the Company's universal, off-the-shelf natural killer cell product candidate derived from a clonal master iPSC line. The clinical trial of FT500 is expected to be the first-ever clinical investigation in USA of an iPSC-derived cell product.

\section{Poseida}

Poseida Therapeutics (CA, USA; https://poseida.com) has received from the FDA a Regenerative Medicine Advanced Therapy (RMAT) designation for P-BCMA-101, Poseida's lead CAR-T therapeutic candidate currently in a Phase I clinical trial for the treatment of patients with relapsed/refractory multiple myeloma. The RMAT designation is a program under the 21 st Century Cures Act that is intended to expedite the development and review of regenerative medicines for the treatment of serious or life-threatening diseases and conditions. A regenerative medicine therapy is eligible for the designation if it is intended to treat, modify, reverse or cure a serious or life-threatening disease or condition, and preliminary clinical evidence indicates that the product has the potential to address unmet medical needs for such a disease or condition. P-BCMA-101 targets cells that express B-cell maturation antigen, or BCMA, which is expressed on essentially all multiple myeloma cells. P-BCMA-101 is engineered with Poseida's nonviral piggyBac ${ }^{\mathrm{TM}}$ DNA Modification System, resulting in a high percentage of $\mathrm{T}$ stem cell memory cells. Preliminary results from the company's ongoing Phase I clinical trial suggest that P-BCMA-101 may have improved response rates with a favorable safety profile compared with published results from clinical trials of other CAR-T therapies at similar doses. Low to no levels of cytokine release syndrome or neurotoxicity have been seen. The Phase I study is funded in part by the California Institute for Regenerative Medicine (CA, USA; www.cirm.ca.gov).

\section{Rocket Pharmaceuticals}

Rocket Pharmaceuticals Inc. (NY, USA; www.rocketpharma.com) has announced the clearance of the Company's IND application for RP-L102, the Company's lentiviral vector-based gene therapy for the treatment of Fanconi Anemia (FA), by the FDA. The clinical trial will evaluate 'Process B' which incorporates higher cell doses, transduction enhancers and commercial-grade vector manufacturing and cell processing. The International Fanconi Anemia Gene Therapy Working Group helped the development of new generation of FA gene therapy programs, which began with an HIV-1-derived, self-inactivating lentiviral vector. RP-L102's lentiviral vector carries the FANC-A gene as part of the PGK-FANCA-WPRE expression cassette, which includes a phosphoglycerate kinase $(P K G)$ promoter and an optimized woodchuck hepatitis virus post-transcriptional regulatory element (WPRE). The ex vivo administration process begins with the removal and isolation of hematopoietic stem cells using a CD34 ${ }^{+}$ selection process. Autologous genetically modified $\mathrm{CD} 34^{+}$enriched hematopoietic cells (fresh or cryopreserved) 
are infused back into patients to restore function. RP-L102 is currently being studied in a Phase I/II clinical trial in the European Union with an Investigational Medicinal Product Dossier in place with the Spanish Agency for Medicines and Health Products. The FDA accepted the Company's IND application for RP-L102 utilizing 'Process B' which incorporates higher cell doses, transduction enhancers and commercial-grade vector. RP-L102 has been granted Orphan Drug designation for the treatment of Fanconi Anemia type A in USA and in Europe.

A few weeks later, the FDA has also granted RMAT and Fast Track designations to RP-L102.

\section{Red light}

Bone Therapeutics

Bone Therapeutics (Belgium; www.bonetherapeutics.com) has announced the conclusions of the Data and Safety Monitoring Board (DSMB) for the interim analysis of the Phase III osteonecrosis study of PREOB in 44 patients with a 12-month follow-up. The DSMB evaluated the results of a formal preplanned interim efficacy analysis in order to assess the efficacy of the injection in the femoral head of 20 million autologous PREOB cells combined with core decompression versus the injection of placebo combined with core decompression, with the intent to stop the study early if there is overwhelming evidence of treatment benefit or futility. The DSMB reported that PREOB was well tolerated by patients. However, the interim results suggest that it is unlikely that the primary objective will be achieved at the final analysis. The DSMB therefore recommended the discontinuation of the trial. As a result, Bone Therapeutics' stock price dropped 25\%. The Company is now focusing on its ALLOB platform and two lead programs:

- Delayed union fractures: the Company has announced in September 2018 positive final results in the Phase I/IIA delayed-union study in 21 patients, supporting the future clinical development of the delayed union indication.

- Spinal fusion: the Company has completed patient recruitment in the Phase IIA spinal fusion study in February 2018. Efficacy and safety data for the full set of 32 patients are expected mid-2019, post a follow-up period of 12 months.

ALLOB, the Company' allogeneic osteoblastic cell therapy product, is derived from ex vivo cultured bone marrow cells of healthy adult volunteer donors, whereas PREOB was an autologous osteoblastic cell therapy product derived from ex vivo cultured bone marrow cells of the patients.

Mergers, acquisitions...

BioTime \& Asterias

BioTime (CA, USA; www.biotimeinc.com) and Asterias Biotherapeutics (CA, USA; www.asteriasbiotherapeutics. com) have entered into a definitive merger agreement whereby BioTime will acquire all of the remaining outstanding common stock of Asterias that are not currently owned by BioTime. Asterias stockholders will receive 0.71 shares of BioTime common shares for every share of Asterias common stock and will own approximately $16.2 \%$ of the combined company.

\section{Capital market \& finances \\ ViaCyte}

ViaCyte (CA, USA; www.viacyte.com) has announced an US\$ 80 million Series D financing led by Bain Capital Life Sciences and joined by TPG and RA Capital Management, as well as existing investor, Sanderling Ventures, and several individual supporters of the Company. Proceeds from the financing will be used to further advance ViaCyte's novel stem cell-derived islet replacement therapies. These therapies have the potential to provide a functional cure for patients with Type 1 diabetes as well as an important option for patients with Type 2 diabetes who depend on insulin to help control their disease. The private financing will be completed in two tranches; further details were not disclosed. Together with the recently announced strategic collaborations and transactions with WL Gore and Associates and CRISPR Therapeutics, ViaCyte has secured commitments for over US\$ 100 million of new financing in the second half of 2018. 


\section{Financial \& competing interests disclosure}

Dusko llic has received an honorarium from Future Science Group for the contribution of this work. The authors have no other relevant affiliations or financial involvement with any organization or entity with a financial interest in or financial conflict with the subject matter or materials discussed in the manuscript apart from those disclosed.

No writing assistance was utilized in the production of this manuscript.

\section{References}

1 Ellebrecht CT, Bhoj VG, Nace A et al. Reengineering chimeric antigen receptor T cells for targeted therapy of autoimmune disease. Science 353(6295), 179-184 (2016). 